\title{
Radical Detection in Harsh Environments by Means of Laser-Induced Fluorescence using a Single Bidirectional Optical Fiber
}

\author{
Heiner Schwarz Robert Schlögl Raimund Horn* \\ Fritz Haber Institute of the Max Planck Society \\ Department of Inorganic Chemistry \\ Faradayweg 4-6, 14195 Berlin, Germany
}

${ }^{*}$ Corresponding author. Email: horn_r@fhi-berlin.mpg.de 


\begin{abstract}
A new experimental method is describe enabling detection of hydroxyl radicals $(\mathrm{OH})$ by LaserInduced Fluorescence (LIF) in high-temperature gasphase reactions. This is accomplished by means of a bidirectional optical fiber probe which is of interest for applications where optical access is limited. An optical setup has been developed which allows simultaneous excitation and detection of fluorescence using one and the same fiber. Caveats resulting form coupling as well as laser-induced scattering are addressed, and different fibers are compared with regard to core material composition and geometric collection efficiency. On this basis a suitable fiber is identified and $\mathrm{OH}$ detection and profile measurements are demonstrated in a premixed laminar flame as reference experiment.
\end{abstract}

\section{Introduction}

Free radicals play a fundamental role in reaction kinetics underlying combustion, plasma chemistry, as well as atmospheric chemistry. Detection of radical species is challenging because of their high reactivity and $\mathrm{ppm}$ to ppb concentrations levels, but might be accomplished by laser-spectroscopic methods such as cavity ring-down absorption (CRDS) or resonance-enhanced multiphoton ionization (REMPI) [14]. A shortcoming of these techniques is the requirement that the measurement has to be performed in an optical cavity in the case of CRDS [22], or the insertion of an ionization probe [2] or a molecular beam nozzle [8] into the reaction medium in REMPI measurements.

Laser-Induced Fluorescence (LIF) is a spectroscopic method that features high sensitivity and potential for in-situ quantification [5, 3]. In LIF the laser wavelength is tuned to a species-specific rovibronic transition of the atom or molecule of interest, which is elevated to an excited electronic state and might - after having undergone collision-induced intra- or intermolecular energy transfer processes - eventually emit a fluorescence photon. The fluorescence signal may be considered proportional to the species' number density if quenching or predissociation effects are accounted for.

In many applications using LIF is hindered by the necessity for optical access to the system. The common right-angle detection arrangement necessitates two optical ports which cannot always be provided. As a representative example one might imagine a reactor the walls of which consist of catalytically active (intransparent) material, a reaction chamber encapsulated by a furnace, or high pressure internal combustion engines. An alternative to direct optical access are optical fibers which can be used for minimally-invasive in-situ detection even under harsh conditions. Common fused silica fibers are chemically inert and show high temperature resistivity. There have been reports of optical fiber probes for (Raman-) measurements comprising distinct fibers for delivering the excitation laser and collecting the scattered radiation [17]. Despite attempts to optimize the probe head design most efficient excitation/collection efficiency is inherently achieved by use of a single bidirectional fiber. Furthermore, the probe heads are rather inadequate for minimally-invasive application under high-temperature conditions. To the knowledge of the authors this is the first demonstration of experiments in which a single optical fiber is used for bidirectional LIF excitation and detection of radical species under harsh conditions.

In what follows we describe LIF detection using an optical fiber probe in a bidirectional manner and demonstrate LIF hydroxyl radical $(\mathrm{OH})$ detection in a a reference experiment. Section 2.1 outlines experimental details of the reference experiment, i.e. the premixed laminar flame, where quantitative $\mathrm{OH}$ concentration have been determined using a combination of conventional $\mathrm{OH}$ LIF and vibrational Raman scattering. Section 2.2 describes the optical setup for simultaneous coupling and detection of laser-induced fluorescence, and discusses complications arising from laser-induced scattering of high-energy laser pulses through optical fibers. Section 3 focusses on the numerical determination of the collection efficiency and the effective detection volume of bidirectional fiber probes based on simple geometric consideration. Results are discussed in Section 4 and first successful fiber probe OH-LIF detection is demonstrated in the premixed laminar flame showing good agreement with the conventional detection scheme.

\section{Experimental}

\subsection{Characterization of Laminar Pre- mixed Flame}

A laminar premixed stoichiometric methane-air flame was used to generate $\mathrm{OH}$ radicals and served as a reference experiment. The atmospheric premixed flame was anchored at the outlet of a $6 \mathrm{~mm}$ tube which could be vertically shifted relative to the optic axis. Axial reference profiles of $\mathrm{OH}$ concentration and temperature along the burner's symmetry axis in the exhaust fume region were measured by means of LIF and vibrational Raman scattering using a conventional lens-type optical setup. Thermodynamic equilibrium considerations allow for quantification of $\mathrm{OH}$ concentrations based on the local temperature in the post-reaction zone.

OH-LIF The hydroxyl radical features electronic transitions in the spectral region around $300 \mathrm{~nm}$ and has thus been excited using a frequency-doubled dye laser (Sirah, Cobra-Strech) which is pumped by the second harmonic of a Q-switched Nd:YAG laser (SpectraPhysics, Quanta-Ray). Typical dye-laser pulses are characterized by a pulse length of $\sim 8 \mathrm{~ns}$, a pulse energy of $\sim 1 \mathrm{~mJ}$, and a nominal fundamental linewidth of 0.06 $\mathrm{cm}^{-1}$. The system was tuned to the $\mathrm{OH} \mathrm{Q}_{1}(8)$ transition of the $\mathrm{A}^{2} \Sigma-\mathrm{X}^{2} \Pi$ (1-0) band at $283.553 \mathrm{~nm}$ (air) which 
shows only a weak temperature-dependance $f_{\mathrm{B}}(T)$ for expected temperatures [19]. Off-resonant detection of the 1-1 and 0-0 bands at 306-325 nm allows spectral separation of elastically scattered light of the excitation laser. In the reference experiment setup (Figure 1 top) the LIF laser is reflected by a longpass dichroic mirror (DM) and focused by a $15 \mathrm{~mm}$ lens L (f-number $f_{\#}=1.5$ ) into the flame detection volume (DV). The fluorescence is collected by the same lens in a collinear manner, and after having passed the dichroic mirror, it is focused onto a fiber bundle coupled to a grating spectrometer (SP). The dispersed light is captured on a conventional CCD without possibility for fast gating (hence the minimal exposure is set by the readout time, $16 \mathrm{~ms}$ ). Typically 1000 individual shots were recorded. The chemiluminescence-corrected (due to the long exposure) LIF intensity may be assumed to be a measure for the $\mathrm{OH}$ number density. The resulting $\mathrm{OH}$ concentration profile above the flame's reaction cone is shown in Figure 2 (blue markers). Quantification is obtained by temperature measurements explained in the section.

Raman Temperature Measurements In measurements conducted by Battles et al. [1] it was shown that local $\mathrm{OH}$ concentrations may be correlated to the local temperature by assuming thermodynamic equilibrium, an assumption valid in the product-gas plume behind the luminescent reaction zone of the flame. In order to calculate the equilibrium composition the local temperature was measured by means of vibrational Raman scattering. Lapp et al. [16, 4] suggest several methods to deduce temperature from vibrational Raman signatures of nitrogen in air-fed flames. The same detection setup as described in the previous paragraph (and shown in Figure 1 top) was thus employed, but a continues-wave $\mathrm{Nd}: \mathrm{YVO}_{4}$ laser (Coherent Verdi V10, $532 \mathrm{~nm}$ ) was used providing a $7 \mathrm{~W}$ beam at right-angles to the collection optics, and $\mathrm{N}_{2}$ spectra were recorded for exposure times of 30 seconds. Using a continuous-wave source under steady-state conditions permits high laser powers to be used without risk of inducing optical breakdown or the need for pulse stretching. The polarization plane could be rotated using a $\lambda / 2$-plate which allows for polarization-separated measurements as proposed by Gruenefeld [6]. Raman scattering occurs predominately perpendicular to the laser's polarization direction. If the laser-polarization is tuned to be parallel to the detection optical axis no Raman signal is captured while unpolarized fluorescence and chemiluminescence is seen (Figure 3 top). The difference spectrum yields the pure Raman signal (Figure 3 bottom). The vibrational Raman temperature was then determined by comparing the area of the $1 \leftarrow 0$ band to the $2 \leftarrow 1$ band using Boltzmann statistics. The integral Raman vibrational band intensity $I_{v}$ (i.e. the sum of all rotational lines within a $v+1 \leftarrow v$ band) is [18]:

$$
I_{v+1 \leftarrow v} \propto \frac{(v+1)}{\lambda_{\mathrm{R}}^{4} Q_{\mathrm{vib}}} \exp \left(-\frac{E(v)}{k_{B} T}\right)
$$
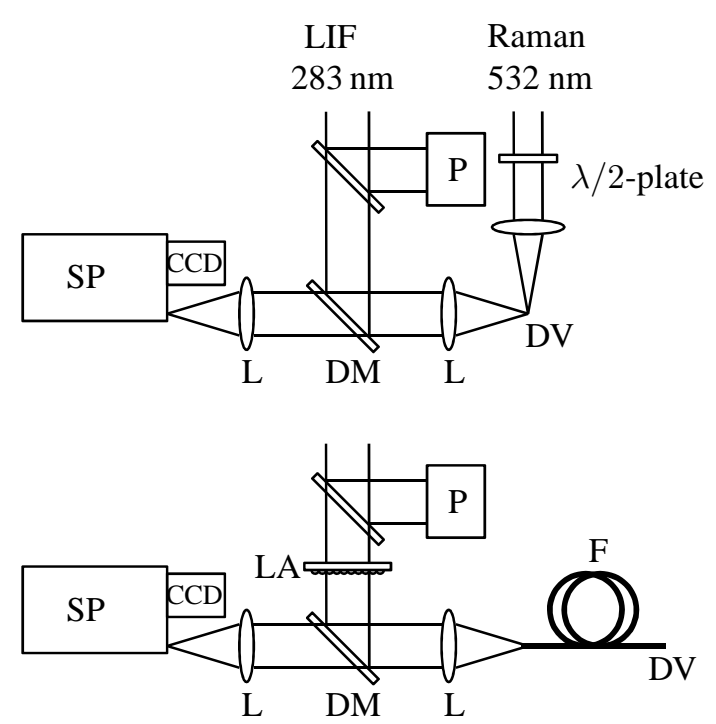

Figure 1: Schematic representation of optical setup: dichroic (longpass) mirror DM, lens L, micro-lens array $\mathrm{LA}$, power-meter $\mathrm{P}$, fiber $\mathrm{F}$, detection volume $\mathrm{DV}$, spectrometer SP.

$v$ being the vibrational quantum number, $\lambda_{\mathrm{R}}$ the Raman wavelength, $Q_{\mathrm{vib}}$ the partition function, and $E(v)$ the molecular energy term.

The resulting temperature profile is also depicted in Figure 2 (red markers) together with the $\mathrm{OH}$ concentration. The experimentally determined temperature maximum of $\sim 2200 \mathrm{~K}$ agrees well with the expected adiabatic flame temperature. Thermodynamic equilibrium calculation were performed using CHEMKIN [13] to obtain local $\mathrm{OH}$ concentrations for calibration.

\subsection{Fiber Coupling and Laser-Induced Scattering}

When optical fibers are used to transmit high power pulsed laser (UV-)radiation coupling, transmission attenuation and inelastic scattering of the fiber material need bo be taken into consideration.

The bottom part of Figure 1 shows the optical setup adapted for bi-directional fiber coupling and detection, which is a modification of the arrangement described in the first section. The coupling lens is chosen such that the NA given by its focal length and the laser diameter corresponds to the numerical aperture NA of the fiber. At the same time, this assures that the fluorescence response returned through the fiber is complectly captured. A tri-axial fiber coupler stage permits positioning of the fiber end-face.

Coupling high-intensity laser-pulses into optical fibers may lead to surface or bulk breakdown processes of the fiber material, such as optical breakdown occurring at the fiber entrance or exit surface [21], as well as re-imaging of the focal point within the fiber leading to bulk damage [20, 7]. Also optical breakdown of the 


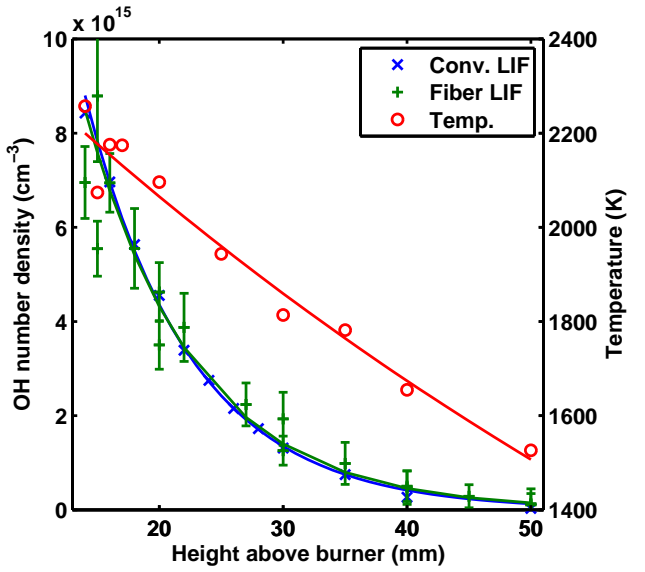

Figure 2: Left axis: $\mathrm{OH}$ number density profiles using conventional lens-type LIF (blue crosses) and fiber probe LIF (green pluses with error bars). Conventional measurement error bars of similar order as for fiber probe but omitted for clarity. Right axis: Raman temperature profile (red circles). Lines are exponential fits.
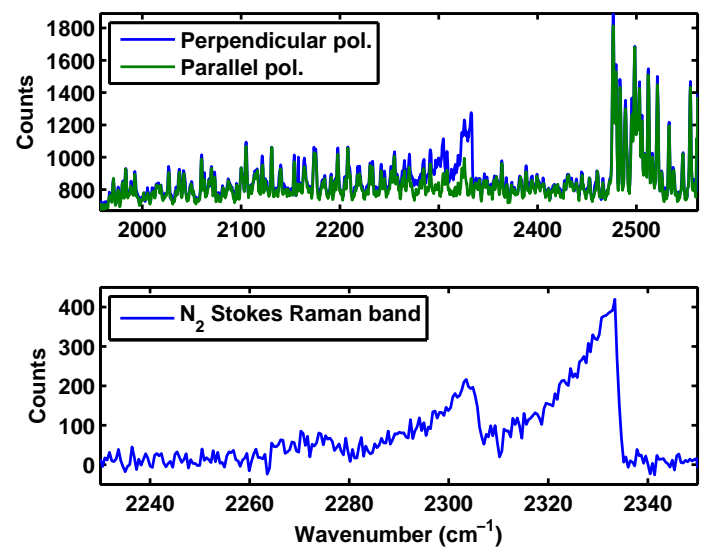

Figure 3: Top: Spectrum with laser polarization perpendicular (including Raman signal) and parallel (only fluorescence and chemiluminescence) to the detection optical axis. Bottom: Difference spectrum featuring the pure Raman signature of $\mathrm{N}_{2}$. Three vibrational bands are perceptible, corresponding to $v=1 \leftarrow 0,2 \leftarrow 1$, and $3 \leftarrow 2$ (from right to left). gas-phase in front of the fiber surface would spoil every spectroscopic investigation, as the waist of the Gaussian beam is of the order of several microns resulting in high peak intensities. With respect to this problem the focal spot intensity distribution was homogenized using a micro-lens array (MA) designed to yield an almost flattop intensity distribution and a focal waist of the size of the fiber diameter [21]. The fiber end-faces are polished using $1 \mu \mathrm{m}$ polishing paper.

Step-index multimode fibers made of fused silica $\left(\mathrm{SiO}_{2}\right)$ with fluorine-doped claddings are commercially available and suitable for UV transmission. They posses high damage threshold intensities, are characterized by their $\mathrm{OH}-$ content (referred to as high-OH and low-OH) and the amount of trace impurities. High-OH fibers show better UV-transmission characteristics than those with low $\mathrm{OH}-$ content. The fiber material's inherent, fabrication-induced impurities and defects may lead to undesired fluorescence, additional Raman bands, colorcenter formation, and photodegradation effects [15, 10, 11, 12]. Stimulated Raman scattering and non-linear effects become important only for longer fibers or higher pump powers [11].

In Figure 4 the back-scattering spectra of two fibers characterized by low and high $\mathrm{OH}$-content are shown. Both samples have the same geometrical properties (core diameter of $500 \mu \mathrm{m}$, length $1 \mathrm{~m}$ ) and comparable pulse-energies are transmitted (indicated in the legend) at a laser wavelength of $280 \mathrm{~nm}$. The Raman spectrum of vitreous fused silica features phonon and defect bands at wavenumbers $<1200 \mathrm{~cm}^{-1}(=290 \mathrm{~nm})$ [15]. These are to a large extent blocked by the dichroic mirror but are still clearly seen for both fiber types. Above $300 \mathrm{~nm}\left(=2400 \mathrm{~cm}^{-1}\right)$ two distinct features are observed. The high-OH fiber has a nearly feature-less spectrum except for a dominant peak around $312 \mathrm{~nm}$ (Raman shift $3714 \mathrm{~cm}^{-1}$, FWHM $112 \mathrm{~cm}^{-1}$ ) which is to be attributed to the vibrational Raman band of the OH-groups [15]. Naturally, the OH Raman peak is redshifted by the same frequency from the excitation wavelength as the $\mathrm{OH}$ fluorescence band $\left(\omega_{e}=3738 \mathrm{~cm}^{-1}\right.$ in the $\mathrm{OH} \mathrm{X} \mathrm{X}^{2} \Pi$ state) and thus interference renders the high-OH fiber less suitable with respect to the detection of $\mathrm{OH}$ molecules. On the other hand the low-OH fiber is characterized by broadband features the origin of which have not been unambiguously identified but might be attributed to fluorescence by trace species originating from the production process or color-center formation upon on high-power UV transmission [11, 12]. It has to be mentioned that scattering depends strongly on accurate coupling alignment indicating that also the fiber cladding may contribute to characteristic spectral features.

The same characteristic fiber scattering spectra (i.e. prominent $\mathrm{OH}$ Raman peak for high-OH fibers, and broadband fluorescence for low-OH fibers) have also been observed for excitation at $355 \mathrm{~nm}$. 


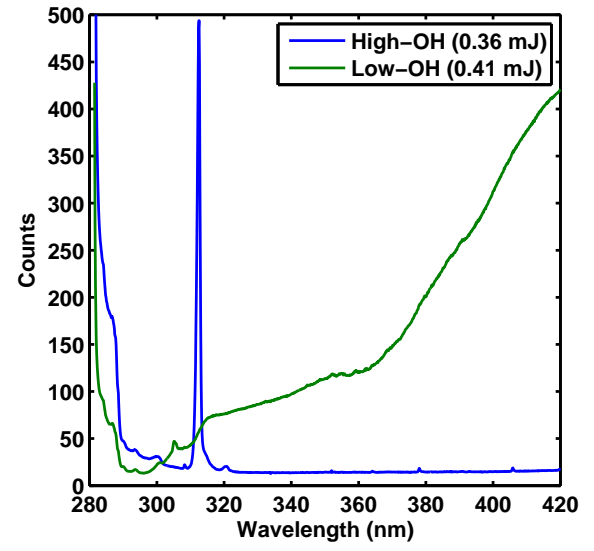

Figure 4: Laser-induced scattering for high- and low$\mathrm{OH}$ fiber (core diameter $500 \mu \mathrm{m}$, length $1 \mathrm{~m}$ ) induced by $280 \mathrm{~nm}$ laser pulses.

\section{Geometrical Considerations on Collection Efficiency}

An expression for the number of fluorescence photons $N_{\text {ph }}$ upon laser-excitation may be given by

$$
N_{\mathrm{ph}}=\frac{B_{12} I_{\nu} \tau}{c} n V f_{\mathrm{B}}(T) A_{21} \tau_{\mathrm{eff}} \frac{\Omega}{4 \pi}
$$

where for simplicity a two-level system is assumed, and $B_{12}$ is the Einstein coefficient of absorption (in $\mathrm{m}^{3} \mathrm{~Hz}$ $\left.\mathrm{J}^{-1} \mathrm{~s}^{-1}\right), I_{\nu}$ the spectral irradiance of the laser (in W $\mathrm{m}^{-2} \mathrm{~Hz}$ ), $\tau$ the pulse length (in s), $c$ the speed of light; $n$ is the number density of the probed molecule (here $\mathrm{OH}$ ) in the electronic ground state (in $\mathrm{m}^{-3}$ ), $V$ the detection volume (in $\mathrm{m}^{3}$ ), $f_{\mathrm{B}}(T)$ the temperature dependant Boltzmann fraction i.e. the portion of molecules in the rovibronic level being excited, $A_{21}=1 / \tau_{\text {rad }}$ Einstein coefficient for spontaneous emission (in $\mathrm{s}^{-1}$ ) with radiative lifetime $\tau_{\text {rad }}, \tau_{\text {eff }}$ (in s) is the effective lifetime of the laser excited state accounting for non-radiative decay (quenching and predissociation), and $\Omega$ the collection solid angle.

Equation (2) holds for an infinitesimal volume $d V$ and hence the total number of fluorescence photons collected for an arbitrary optical system is found by spatial integration

$$
N_{\mathrm{ph}}=\int_{V} d N_{\mathrm{ph}}=\int_{V} \frac{\partial N_{\mathrm{ph}}}{\partial V} d V
$$

where $\partial N_{\mathrm{ph}} / \partial V=B I \tau c^{-1} n f_{\mathrm{B}} A \tau_{\text {eff }}(\Omega / 4 \pi)$ is readily found from Equation (2). In a homogenous environment the only parameters showing a spatial dependance are $I(\vec{x})$ and $\Omega(\vec{x}) / 4 \pi$ if absorption is neglected for the time being. It is convenient to assume cylindrical coordinates $\vec{x}(r, \varphi, z)$ so that we can define the detection efficiency

$$
\Gamma:=\int_{V} I(\vec{x}) \frac{\Omega(\vec{x})}{4 \pi} d \varphi r d r d z
$$

as a quantitative measure for the number of collected photons for different optical setups (for convenience we only consider the irradiance $I$ instead of the spectral quantity $I_{\nu}$ ).

On the one hand it is desirable to predict the detection efficiency's dependance on fiber parameters (like fiber diameter or numerical aperture), as well as to assess the size of the detection volume and the spatial resolution. On the other hand one would like to compare quantitatively the detection efficiency of an optical fiber probe to a conventional lens-type optical setup. The following section outlines an approach to describe the irradiance distribution $I(\vec{x})$, and the detection solid angle field $\Omega(\vec{x})$ of an optical fiber and to determine numerically the collection efficiency $\Gamma$ based on the general expression Equation (2) for the fiber probe.

Fiber Probe Collection Efficiency A first-order estimate for the radiation field $I(\vec{x})$ behind the fiber endface is found by assuming the light emanates from a point source (i.e. $I(\vec{x})=C / \vec{x}^{2}$ ) and is isotropically distributed over the solid angle defined by the numerical aperture $\Theta_{\mathrm{NA}}=\arcsin (\mathrm{NA})$. According to Gauss' divergence theorem integrating $I(\vec{x})$ over a closed surface yields the total radiative power $P$ within the volume enclosed by the integration surface, i.e. $P=\int I(\vec{x}) d S$. After integration over $\Theta_{\mathrm{NA}}$ the constant is found to be $C=P / 2 \pi\left(1-\cos \theta_{\mathrm{NA}}\right)$ so that the radiation field can be readily expressed as

$$
I(\vec{x})=\frac{P}{2 \pi\left(1-\cos \theta_{\mathrm{NA}}\right) \vec{x}^{2}} .
$$

Determining the effective collection solid angle $\Omega(\vec{x})$ of the fiber is a purely geometrical problem. The solid angle of an arbitrary surface $S$ seen from a point $\vec{x}$ in space may be defined as

$$
\Omega(\vec{x})=\int_{S\left(\overrightarrow{x^{\prime}}\right)} \frac{\vec{X} \cdot d \overrightarrow{x^{\prime}}}{X^{3}} .
$$

The integration is performed over $S\left(\overrightarrow{x^{\prime}}\right)$ with $d \overrightarrow{x^{\prime}}=$ $\vec{n}_{S} d S\left(\overrightarrow{x^{\prime}}\right)$ normal to $S\left(\overrightarrow{x^{\prime}}\right)$ and $\vec{X}=\overrightarrow{x^{\prime}}-\vec{x}$. In the present consideration the surface $S$ corresponds to the fiber end-face.

Optical rays impinging on the surface at an angle which is greater than the critical acceptance angle of the optical fiber $\theta_{\mathrm{NA}}=\arcsin (\mathrm{NA})$ will be reflected and not contribute to the effective collection solid angle. In mathematical terms this condition is readily expressed in terms of the angle $\angle\left(\vec{X}, d \overrightarrow{x^{\prime}}\right)$, i.e. only if $L\left(\vec{X}, d \overrightarrow{x^{\prime}}\right)<\theta_{\mathrm{NA}}$ does the surface element $d \overrightarrow{x^{\prime}}$ contribute to the integration. Thus, we may rewrite Equation (6) as a conditional integral:

$$
\begin{gathered}
\Omega(\vec{x})=\int_{S} \frac{\vec{X} \cdot d \overrightarrow{x^{\prime}}}{X^{3}} . \\
\angle\left(\vec{X}, d \overrightarrow{x^{\prime}}\right)<\theta_{\mathrm{NA}}
\end{gathered}
$$




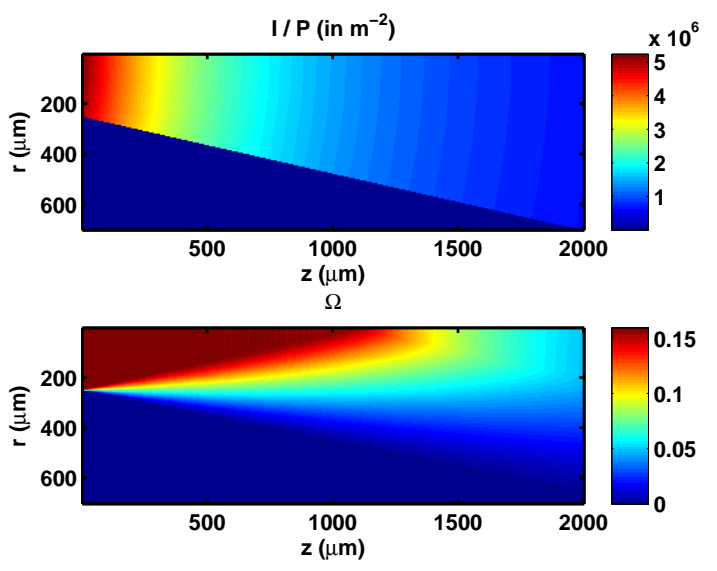

Figure 5: Irradiance field $I(\vec{x})$ (top), and effective collection solid angle distribution $\Omega(\vec{x})$ (bottom) in cylindrical coordinates (radial coordinated $r$ and axial coordinated $z$. Fiber parameters: numerical aperture NA $=$ 0.22 and diameter $D_{\text {fiber }}=500 \mu \mathrm{m}$.

Knowledge of $I(\vec{x})$ and $\Omega(\vec{x})$ (Figure 5) now allows for determining the effective detection volume and the collection efficiency $\Gamma$ by means of evaluating integral (4).

Conventional Lens-Type Collinear Detection It is of interest to compare the above result to the collection efficiency for a conventional LIF arrangement such as the lens-type collinear setup presented in the prior Section 2.1. This may be accomplished by considering that the collection solid angle is essentially constant over the detection volume seen by the collection lense (i.e. $\Omega(\vec{x})=\Omega_{0}=$ const) so that

$$
\begin{aligned}
& \int_{V} I(r, z) \frac{\Omega_{0}}{4 \pi} d \varphi r d r d z= \\
& \frac{\Omega_{0}}{4 \pi} \int_{z=-a f / D_{\text {lens }}}^{a f / D_{\text {lens }}} \int_{r=0}^{\infty} \int_{\varphi=0}^{2 \pi} I(r, z) d \varphi r d r d z .
\end{aligned}
$$

The inner two integrals corresponds to the radiant power $P$ which is constant along the optical axis $z$, and the integration along $z$ may as a first order estimate be performed over the depth of field $2 a f / D_{\text {lens }}$ ( $a$ detection aperture).

\section{Results}

\subsection{Collection Efficiency and Detection Volume of Bidirectional Fiber}

In Section 3 the fiber's excitation and detection distribution were determined, shown in Figure 5] so that the resulting detection efficiency $\Gamma$ may be evaluated for different fiber geometries. It turns out that $\Gamma / P$ (normalized by the total radiant power $P$ ) amounts to $\sim 1 \times 10^{-5} \mathrm{~m}$ for the fiber dimensions used in experiments presented here $\left(\mathrm{NA}=0.22\right.$ and diameter $D_{\text {fiber }}=$ $500 \mu \mathrm{m})$. It is of interest to compare this value to the collection efficiency for a conventional LIF arrangement such as the collinear setup presented in Section 2.1 This results in $\Gamma / P=4 \times 10^{-5} \mathrm{~m}$ for the presented optical setup. This implies that we can expect LIF intensities of the same order of magnitude for the lens-type LIF reference experiment as for the fiber probe measurements, the latter having a slightly smaller photon count. This is confirmed by results presented below.

Evaluation of integral (4) further yields that $50 \%$ of the LIF signal is collected from an area within a distance of $1.0 \mathrm{~mm}$ from the fiber endface. This corresponds roughly to the length of the cone of maximum collection solid angle given by $D / 2 \tan \theta_{\mathrm{NA}}=1.1 \mathrm{~mm}$. At the same time $90 \%$ of the LIF photons are collected from an area within $6.4 \mathrm{~mm}$ distance from the fiber face. The detection volume and the spatial resolution is hence in the range of several $\mathrm{mm}$ and scales as the fiber diameter. Calculations have also shown that the collection efficiency $\Gamma$ scales linearly with increasing fiber diameter as well as typical numerical apertures NA (multimode step index fiber (MSIF) with non-silica cladding $\mathrm{NA}=0.37$, all-silica MSIF NA $=0.22$, single-mode fiber $(\mathrm{SMF}) \mathrm{NA}=0.13$, and large mode-area photonic crystal fiber $(\mathrm{PCF}) \mathrm{NA}=0.04)$.

Apart from the fiber diameter's influence on collection efficiency one has to consider that thinner fibers permit less transmitted radiant power $P$ due to the core material's threshold damage. Hence, it can be expected that $P$ is roughly inversely proportional to the square of the fiber diameter $D_{\text {fiber }}$. This leads to the conclusion that the number of detectable fluorescence photons $N_{\mathrm{ph}} \propto P \Gamma$ scales as $D^{3}$. This trend is confirmed by including results of the measurements conducted by Hsu et al. [9] who have investigated damage thresholds of various fiber types and sizes (Table 1 ). The $D^{3}$ scaling is confirmed for fibers of which different sizes have been investigated, i.e. the multi-mode fibers. It can also be seen that single-mode and large mode-area photonic crystal fibers, which are both characterized by a small (mode-)core diameter, are less suitable due to their small collection efficiency as well as attainable pulse energy.

\subsection{LIF OH Detection using Bidirectional Fiber Probe in Harsh Environment}

In Section 2.1 measurements of the $\mathrm{OH}$ concentration and temperature profiles in a stoichiometric methane-air flame were outlined. OH LIF was also performed using the fiber probe technique described above by traversing the fiber endface perpendicular to the flames's symmetry axis. Figure 6 shows the OH LIF spectrum $\left(\mathrm{A}^{2} \Sigma\right.$ $\mathrm{X}^{2} \Pi 1 \leftarrow 1$ and $0 \leftarrow 1$ band) for the two LIF arrangements, as well as the non-resonant fiber scattering background. Three conclusions may be drawn: first, the $\mathrm{OH}$ LIF spectrum is unaffected by the presence of the fiber 


\begin{tabular}{lrrrrr}
\hline Fiber type & $\begin{array}{r}\text { Diameter } \\
(\mu \mathrm{m})\end{array}$ & NA & $\begin{array}{r}E_{\text {trans }} \\
(\mathrm{mJ})\end{array}$ & $\begin{array}{r}\Gamma / P \\
\left(10^{5} \mathrm{~m}\right)\end{array}$ & $\begin{array}{r}E_{\text {trans }} \Gamma / P \\
\left(10^{5} \mathrm{~mJ} \mathrm{~m}\right)\end{array}$ \\
\hline LMA-PCF & 15 & 0.04 & 0.004 & 0.007 & 0.00003 \\
SMF & 3.5 & 0.13 & 0.002 & 0.005 & 0.00001 \\
All-silica MSIF & 200 & 0.22 & 0.5 & 0.5 & 0.3 \\
All-silica MSIF & 550 & 0.22 & 2.2 & 1.4 & 3.1 \\
MSIF & 400 & 0.37 & 0.5 & 1.7 & 0.9 \\
MSIF & 600 & 0.37 & 1.5 & 2.6 & 3.9 \\
MSIF & 800 & 0.37 & 2.9 & 3.5 & 10.1 \\
MSIF & 1000 & 0.37 & 3.9 & 4.3 & 16.9 \\
\hline
\end{tabular}

Table 1: Threshold transmitted pulse energy and overall collection efficiency for various fiber types and sizes. Data for $E_{\text {trans }}$ corresponds to 150 ps pulses at $532 \mathrm{~nm}$ (adapted from [9]).

probe meaning that quenching effects are not observed in the spectrum; second, the inelastic fiber scattering, which was the same when the fiber was inside or outside the flame, is exceeded by the OH LIF signal; third, one finds a comparable LIF signal intensity - the conventional LIF spectrum being scaled by factor of $1 / 2$ in the figure - for both optical arrangements as predicted by the above collection efficiency considerations.

The $\mathrm{OH}$ concentration gradient was scanned using the fiber probe, the outcome of which is included in Figure 2. The $\mathrm{OH}$ profile is resolved and in good agreement with the reference experiment. The relatively large error bars of the LIF measurements shown for the fiber in Figure 2 (conventional measurement error bars of similar order but omitted for clarity) are due to flame fluctuations and shot-to-shot fluctuations of the laser which could not be corrected for by the averaging power-meter used.

At first glance, it might be surprising that the fiber withstands the excessive flame temperatures. This may be explained by assuming an equilibrium between convective heat transfer $\dot{Q}_{\mathrm{conv}}=\alpha A\left(T-T_{\text {gas }}\right)$ from the hot product gases to the fiber, and heat losses due to conduction $\dot{Q}_{\text {cond }}$ and radiative cooling $\dot{Q}_{\mathrm{rad}}=$ $\varepsilon \sigma A\left(T^{4}-T_{0}^{4}\right)(\sigma$ is the Stefan-Boltzmann constant). An emissivity of 0.9 has been assumed for fused silica, and the heat transfer coefficient $\alpha$ has been evaluated from a Nusselt number correlation of the flow field (cylinder in cross flow). It turns out that conduction losses are negligible and that an equilibrium temperature of $T \sim 1400 \mathrm{~K}$ results. Nevertheless the physical intrusion of the fiber close to the reaction zone disturbs the flow field and thus the flame cone which causes slightly biased results at low heights.

\section{Conclusion}

Detection of $\mathrm{OH}$ LIF has been demonstrated using a bidirectional fiber probe technique with the potential for minimally-invasive measurement under harsh conditions. Reference experiments have been performed (OH-LIF and Raman) in order to characterize a stoichiometric methane-air flame serving as a reference

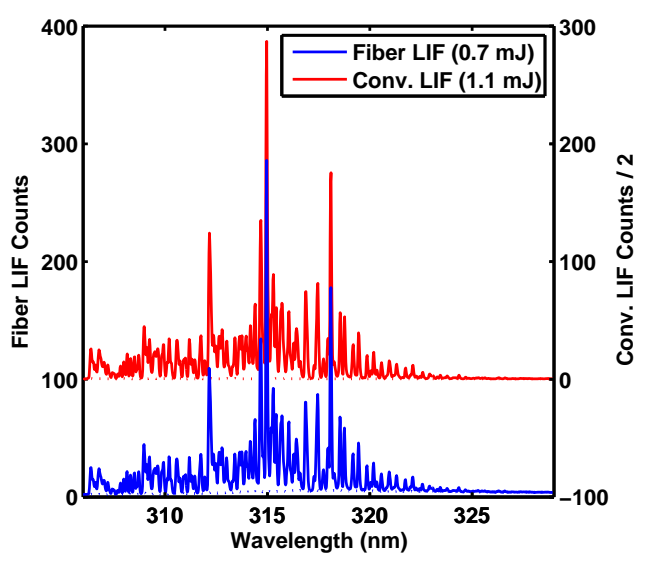

Figure 6: OH LIF spectrum of fiber probe and conventional lens-type optical setup LIF spectrum (scaled by a factor of $1 / 2$ and shifted for clarity). The dashed line is the background including chemiluminescence and fiber scattering. For fiber probe LIF the low-OH fiber is used.

experiment. An optical setup has been presented which permits simultaneous coupling into the fiber and detection of the backscattered fluorescence signal. The detection efficiency and the dimensions of the sample volume have been characterized numerically using geometrical considerations. All-silica optical fibers may be found which are suitable for UV-radiation transmission and allow detection of fluorescence, which is red-shifted beyond the vibrational Raman signature of the fiber $\left(>1000 \mathrm{~cm}^{-1}\right)$. OH LIF was detected and the $\mathrm{OH}$ concentration profile in a laminar premixed flame could be reproduced.

\section{Acknowledgements}

The authors kindly appreciate the financial support granted by the "Cluster of Excellence Unifying Concepts in Catalysis" (UniCat), Berlin. Furthermore, we would like to express our thanks to Gerard Meijer for his scientific support and helpful discussions. 


\section{References}

[1] Brett E. Battles and Ronald K. Hanson. Laserinduced fluorescence measurements of no and oh mole fraction in fuel-lean, high-pressure (1-10 atm) methane flames: Fluorescence modeling and experimental validation. Journal of Quantitative Spectroscopy and Radiative Transfer, 54(3):521537, 1995.

[2] Terrill A. Cool. Quantitative measurement of no density by resonance three-photon ionization. Applied optics, 23(10):1559 EP - 1572, 1984.

[3] J. W. Daily. Laser induced fluorescence spectroscopy in flames. Progress in Energy and Combustion Science, 23(2):133-199, 1997.

[4] M. C. Drake, M. Lapp, and C. M. Penney. Use of the vibrational raman effect for gas temperature measurements. In James F. Schooley, editor, Temperature. American Inst. of Physics, New York, 1982.

[5] Alan C. Eckbreth. Laser diagnostics for combustion temperature and species, volume 7 of Energy and engineering science series. Abacus Press, Tunbridge Wells, 1988.

[6] G. Gruenefeld, V. Beushausen, and P. Andresen. Interference-free uv-laser-induced raman and rayleigh measurements in hydrocarbon combustion using polarization properties. Applied physics. B, Lasers and optics, B61(5):473 EP 478, 1995.

[7] Duncan P Hand, Jonathan D Entwistle, Robert R J Maier, Andreas Kuhn, Clive A Greated, and Julian D C Jones. Fibre optic beam delivery system for high peak power laser piv illumination. Measurement Science and Technology, 10(3):239, 1999.

[8] N. Hansen, T. A. Cool, P. R. Westmoreland, and K. Kohse-Hoinghaus. Recent contributions of flame-sampling molecular-beam mass spectrometry to a fundamental understanding of combustion chemistry. PROGRESS IN ENERGY AND COMBUSTION SCIENCE, 35(2):168-191, 2009.

[9] Paul Hsu, Anil Patnaik, James Gord, Terrence Meyer, Waruna Kulatilaka, and Sukesh Roy. Investigation of optical fibers for coherent antistokes raman scattering (cars) spectroscopy in reacting flows. Experiments in Fluids, pages 1-16, 2010.

[10] Hiroaki Imai and Hiroshi Hirashima. Intrinsic- and extrinsic-defect formation in silica glasses by radiation: Proceedings of the first pac rim meeting on glass and optical materials. Journal of NonCrystalline Solids, 179(0):202-213, 1994.
[11] P. Karlitschek, G. Hillrichs, and K. F Klein. Photodegradation and nonlinear effects in optical fibers induced by pulsed uv-laser radiation. Optics Communications, 116(1-3):219-230, 1995.

[12] P. Karlitschek, G. Hillrichs, and K. F Klein. Influence of hydrogen on the colour center formation in optical fibers induced by pulsed uv-laser radiation.: Part 2: All-silica fibers with low-oh undoped core. Optics Communications, 155(4-6):386-397, 1998.

[13] R. J. Kee, F. M. Rupley, J. A. Miller, M. E. Coltrin, J. F. Grcar, E. Meeks, H. K. Moffat, A. E. Lutz, G. Dixon-Lewis, M. D. Smooke, J. Warnatz, G. H. Evans, R. S. Larson, R. E. Mitchell, L. R. Petzold, W. C. Reynolds, M. Caracotsios, W. E. Stewart, P. Glarborg, C. Wang, C. L. McLellan, O. Adigun, W. G. Houf, C. P. Chou, S. F. Miller, P. Ho, P. D. Young, D. J. Young, D. W. Hodgson, M. V. Petrova, and K. V. Puduppakkam. Chemkin 15101, 2011.

[14] Katharina Kohse-Höinghaus and Jay Barker Jeffries. Applied combustion diagnostics. Combustion. Taylor \& Francis, New York, 2002.

[15] Guey-Liou Lan, P. K. Banerjee, and S. S. Mitra. Raman scattering in optical fibers. Journal of Raman Spectroscopy, 11(5):416-423, 1981.

[16] M. Lapp, L. Goldman, and C. Penney. Raman scattering in flames. Science, 175(4026), 1972.

[17] Ian R. Lewis and Peter R. Griffiths. Raman spectrometry with fiber-optic sampling. Appl. Spectrosc., 50(10):12A-30A, 1996.

[18] Derek Albert Long. Raman spectroscopy. McGraw-Hill, New York, 1977.

[19] J. Luque and D. R. Crosley. Lifbase: Database and spectral simulation (version 1.5). SRI International Report, (MP 99-009), 1999.

[20] B. Richou and P. PellatFinet. Delivery of ndyag laser pulses by large optical fiber: Dependence of the laser intensity profile on threshold of energy transmission. HIGH-POWER LASERS: APPLICATIONS AND EMERGING APPLICATIONS, 2789:186 EP - 194, 1996.

[21] T. Schmidt Uhlig, P. Karlitschek, G. Marowsky, and Y. Sano. New simplified coupling scheme for the delivery of $20 \mathrm{mw}$ nd:yag laser pulses by large core optical fibers: Yag laser pulses by large core optical fibers. Applied physics. B, Lasers and optics, 72(2):183 EP - 186, 2001.

[22] Alexander Schocker, Katharina Kohse-Höinghaus, and Andreas Brockhinke. Quantitative determination of combustion intermediates with cavity ringdown spectroscopy: systematic study in propene 
flames near the soot-formation limit. Appl. Opt., 44(31):6660-6672, 2005. 